TRANSACTIONS OF THE

AMERICAN MATHEMATICAL SOCIETY

Volume 351, Number 1, January 1999, Pages 285-306

S 0002-9947(99)01981-9

\title{
EXISTENCE AND UNIQUENESS FOR A DEGENERATE PARABOLIC EQUATION WITH $L^{1}$-DATA
}

\author{
F. ANDREU, J. M. MAZÓN, S. SEGURA DE LEÓN, AND J. TOLEDO
}

ABstract. In this paper we study existence and uniqueness of solutions for the boundary-value problem, with initial datum in $L^{1}(\Omega)$,

$$
\begin{gathered}
u_{t}=\operatorname{div} \mathbf{a}(x, D u) \quad \text { in }(0, \infty) \times \Omega, \\
-\frac{\partial u}{\partial \eta_{a}} \in \beta(u) \text { on }(0, \infty) \times \partial \Omega, \\
u(x, 0)=u_{0}(x) \quad \text { in } \Omega,
\end{gathered}
$$

where $\mathbf{a}$ is a Carathéodory function satisfying the classical Leray-Lions hypothesis, $\partial / \partial \eta_{a}$ is the Neumann boundary operator associated to a, $D u$ the gradient of $u$ and $\beta$ is a maximal monotone graph in $\mathbb{R} \times \mathbb{R}$ with $0 \in \beta(0)$.

\section{INTRODUCTION}

This paper is devoted to the solvability of the nonlinear parabolic equation

$$
u_{t}=\operatorname{div} \mathbf{a}(x, D u)
$$

with initial data in $L^{1}(\Omega), \Omega$ being a domain in $\mathbb{R}^{N}$ (bounded or unbounded) and a a Carathéodory function satisfying the classical Leray-Lions hypothesis, i.e., $\mathbf{a}$ is a vector valued function mapping $\Omega \times \mathbb{R}^{N}$ into $\mathbb{R}^{N}$ and satisfying

$\left(\mathrm{H}_{1}\right) \mathbf{a}$ is a Carathéodory function (i.e., the map $\xi \rightarrow \mathbf{a}(x, \xi)$ is continuous for almost all $x$ and the map $x \rightarrow \mathbf{a}(x, \xi)$ is measurable for every $\xi)$ and there exists $\lambda>0$ such that

$$
\langle\mathbf{a}(x, \xi), \xi\rangle \geq \lambda|\xi|^{p} \quad(1<p<\infty)
$$

for every $\xi$ and a.e. $x \in \Omega$, where $\langle$,$\rangle is the scalar product in \mathbb{R}^{N}$. There is no restriction in assuming that $\lambda=1$.

$\left(\mathrm{H}_{2}\right)$ For every $\xi$ and $\eta \in \mathbb{R}^{N}, \xi \neq \eta$, and a.e. $x \in \Omega$,

$$
\langle\mathbf{a}(x, \xi)-\mathbf{a}(x, \eta), \xi-\eta\rangle>0 .
$$

$\left(\mathrm{H}_{3}\right)$ There exists $\Lambda \in \mathbb{R}$ such that

$$
|\mathbf{a}(x, \xi)| \leq \Lambda\left(j(x)+|\xi|^{p-1}\right)
$$

for every $\xi \in \mathbb{R}^{N}$ with $j \in L^{p^{\prime}}, p^{\prime}=p /(p-1)$.

Received by the editors September 11, 1995 and, in revised form, December 2, 1996.

1991 Mathematics Subject Classification. Primary 35K65, 47H20.

Key words and phrases. Non-linear parabolic equations, non-linear boundary conditions, $p$ Laplacian, accretive operators, mild solutions.

This research has been partially supported by DGICYT, Project PB94-0960.

(C)1999 American Mathematical Society 
The hypotheses $\left(\mathrm{H}_{1}\right),\left(\mathrm{H}_{2}\right)$ and $\left(\mathrm{H}_{3}\right)$ are classical in the study of nonlinear operators in divergence form (see $[\mathrm{L}])$. The model example of a function a satisfying these hypothesis is $\mathbf{a}(x, \xi)=|\xi|^{p-2} \xi$. The corresponding operator is the $p$-Laplacian operator $\Delta_{p}(u)=\operatorname{div}\left(|D u|^{p-2} D u\right)$. This operator has been widely considered in the literature of PDE. It represents one of the simpler examples of degenerate nonlinear operators for which the classical theory is not available. It also appears in several physical problems - for instance, in non-Newtonian fluids (see [DH] and the literature cited therein).

Recently, in $[\mathrm{B}-\mathrm{V}]$, a new concept of solution has been introduced for the elliptic equation

$$
\begin{gathered}
-\operatorname{div} \mathbf{a}(x, D u)=f(x) \text { in } \Omega, \\
u=0 \quad \text { on } \partial \Omega,
\end{gathered}
$$

namely entropy solution. As a consequence, an $m$-completely accretive operator in $L^{1}(\Omega)$ can be associated to the corresponding parabolic equation. In [AMST], using the method developed in $[\mathrm{B}-\mathrm{V}]$, we study entropy solutions for the elliptic problem with non-linear boundary conditions. Precisely, we study existence and uniqueness of entropy solutions for equations of the form

$$
\begin{gathered}
u-\operatorname{div} \mathbf{a}(x, D u)=f \quad \text { in } \Omega, \\
-\frac{\partial u}{\partial \eta_{a}} \in \beta(u) \quad \text { on } \partial \Omega,
\end{gathered}
$$

where $\partial / \partial \eta_{a}$ is the Neumann boundary operator associated to a, i.e.,

$$
\frac{\partial u}{\partial \eta_{a}}:=\langle\mathbf{a}(x, D u), \eta\rangle
$$

with $\eta$ the unit outward normal on $\partial \Omega, D u$ the gradient of $u$ and $\beta$ a maximal monotone graph in $\mathbb{R} \times \mathbb{R}$ with $0 \in \beta(0)$. These nonlinear fluxes on the boundary occur in heat transfer between solids and gases (cf. [Fr]) and in some problems in mechanics and physics [DL] (see also [Br-2]). Observe also that the classical Neumann and Dirichlet boundary conditions correspond to $\beta=\mathbb{R} \times\{0\}$ and $\beta=$ $\{0\} \times \mathbb{R}$, respectively.

As a consequence of the results of $[\mathrm{B}-\mathrm{V}]$ and $[\mathrm{AMST}]$, we can solve the equation (E) (with non-linear boundary conditions in case $\Omega$ is bounded and with Dirichlet boundary conditions in case $\Omega$ is unbounded) from the point of view of nonlinear semigroups theory, i.e., for every initial datum in $L^{1}(\Omega)$, there exists a unique mild solution given by the Crandall-Liggett exponential formula. However, in principle, it is not clear how these mild solutions have to be interpreted. The purpose of the present paper is to characterize these mild solutions, for which the problem is well posed, by introducing a new class of weak solutions, namely entropy solutions. More precisely, we prove that mild solutions and entropy solutions coincide.

The study of the Cauchy problem for equations of type (E) has received a great deal of attention. For example, existence of weak solutions with measures as initial data, in the case of $\Omega$ bounded with Dirichlet boundary conditions, has been studied in [BG-2], [Ra-1] and [Ra-2]. For some results about existence of weak solutions of similar equations with non-linear boundary conditions see $[\mathrm{X}]$. With respect to existence-uniqueness results for Cauchy problems of type (E), we only know the one given by E. Di Benedetto and M. A. Herrero in [DiH-1] and [DiH-2] (see also [Di-1] and [Di-2]) for the $p$-Laplacian equation in $\mathbb{R}^{N}$. In this case, they introduce a 
class of weak solutions and prove existence and uniqueness of this type of solutions when the initial datum is positive. The non-negativity of the initial data and the homogeneity of the $p$-Laplacian are essential in their proof of uniqueness, since they use some sort of time-compactness via the regularizing effect of Bénilan-Crandall [BCr-1]. Di Benedetto in [Di-2] says the following: "It would be of interest to have a notion of solution that is irrespective of the sign of the solution and a correspondent existence-uniqueness theorem. We remark that the problem is open even if one considers the boundary value problem in bounded domain". The aim of this paper is to answer this question.

The plan of the paper is as follows: Some preliminary results and notation are collected in Section 2. In the third section we study the case of bounded $\Omega$. We prove existence and uniqueness results for the entropy solution of the initial-value problem for equation (E) with non-linear boundary condition, and we show that the entropy solution coincides with the mild solution. In the last section we establish results similar to those of the previous section for the case of Dirichlet boundary conditions and $\Omega$ not necessarily bounded.

\section{Preliminaries}

In this section we give some of the notation and definitions used later. If $\Omega \subset \mathbb{R}^{N}$ is a Lebesgue measurable set, $\lambda_{N}(\Omega)$ denotes its measure. The norm in $L^{p}(\Omega)$ is denoted by $\|\cdot\|_{p}, 1 \leq p \leq \infty$. If $k \geq 0$ is an integer and $1 \leq p \leq \infty, W^{k, p}(\Omega)$ is the Sobolev space of functions $u$ on the open set $\Omega \subset \mathbb{R}^{N}$ for which $D^{\alpha} u$ belongs to $L^{p}(\Omega)$ when $|\alpha| \leq k$, with its usual norm $\|\cdot\|_{k, p} . W_{0}^{k, p}(\Omega)$ is the closure of $\mathcal{D}(\Omega)=C_{0}^{\infty}(\Omega)$ in $W^{k, p}(\Omega)$. For vector-valued functions we follow the notation and definitions of [Br-2]. For instance, if $X$ is a Banach space, $a<b$ and $1 \leq p \leq \infty$, $L^{p}(a, b ; X)$ denotes the space of all measurable functions $u:[a, b] \rightarrow X$ such that $\|u(s)\|$ belongs to $L^{p}([a, b])$. If $v \in L^{1}(\Omega)$ and $\lambda_{N}(\Omega)<\infty$, we denote by $\bar{v}$ the average of $v$, i.e.,

$$
\bar{v}:=\frac{1}{\lambda_{N}(\Omega)} \int_{\Omega} v(x) d x .
$$

Given a finite measure space $(S, \nu)$, we denote by $M(S, \nu)$ the set of all measurable functions $u: S \rightarrow \mathbb{R}$ finite a.e., identifying the functions that are equal a.e.

We recall, cf. [BBC], that for $0<q<\infty$ the Marcinkiewicz space $M^{q}(\Omega)$ can be defined as the set of measurable functions $f: \Omega \rightarrow \mathbb{R}$ such that the corresponding distribution function

$$
\phi_{f}(k)=\lambda_{N}\{x \in \Omega:|f(x)|>k\}
$$

satisfies an estimate of the form

$$
\phi_{f}(k) \leq C k^{-q}, \quad C<\infty .
$$

For bounded $\Omega$ 's, it is immediate that $M^{q}(\Omega) \subset M^{\hat{q}}(\Omega)$ if $\hat{q} \leq q$; also $L^{q}(\Omega) \subset$ $M^{q}(\Omega) \subset L^{r}(\Omega)$ if $1 \leq r<q$.

We will use the following truncation operator: For a given constant $k>0$ we define the cut function $T_{k}: \mathbb{R} \rightarrow \mathbb{R}$ as

$$
T_{k}(s):= \begin{cases}s & \text { if }|s| \leq k, \\ k \operatorname{sign}(s) & \text { if }|s|>k .\end{cases}
$$


For a function $u=u(x), x \in \Omega$, we define the truncated function $T_{k} u$ pointwise, i.e., for every $x \in \Omega$ the value of $T_{k} u$ at $x$ is just $T_{k}(u(x))$. Observe that

$$
\lim _{k \rightarrow 0} \frac{1}{k} T_{k}(s)=\operatorname{sign}(s):= \begin{cases}1 & \text { if } s>0 \\ 0 & \text { if } s=0, \\ -1 & \text { if } s<0 .\end{cases}
$$

By the Stampacchia Theorem, cf. $[\mathrm{KS}]$, if $u \in W^{1,1}(\Omega)$, we have

$$
D T_{k}(u)=1_{\{|u|<k\}} D u,
$$

where $1_{B}$ denotes the characteristic function of a measurable set $B \subset \Omega$.

We need to define the trace of functions which are not in the Sobolev spaces. Before discussing this concept of trace we recall the following spaces introduced in $[\mathrm{B}-\mathrm{V}]: \mathcal{T}_{\text {loc }}^{1,1}(\Omega)$ is defined as the set of measurable functions $u: \Omega \rightarrow \mathbb{R}$ such that for every $k>0$ the truncated function $T_{k}(u)$ belongs to $W_{l o c}^{1,1}(\Omega)$. For $1<p<\infty$, $\mathcal{T}_{\text {loc }}^{1, p}(\Omega)$ is the subset of $\mathcal{T}_{\text {loc }}^{1,1}(\Omega)$ consisting of the functions $u$ such that $D T_{k}(u) \in$ $L_{\text {loc }}^{p}(\Omega)$ for every $k>0$. Likewise, $\mathcal{T}^{1, p}(\Omega)$ is the subset of $\mathcal{T}_{\text {loc }}^{1,1}(\Omega)$ consisting of the functions $u$ such that $D T_{k}(u) \in L^{p}(\Omega)$ for every $k>0$. Observe that in the definition of $\mathcal{T}^{1, p}(\Omega)$ the condition $T_{k}(u) \in L^{p}(\Omega)$ is not imposed. Of course, this condition follows immediately when $\Omega$ is bounded. So, if $\Omega$ is bounded, we have

$$
\mathcal{T}^{1, p}(\Omega)=\left\{u: \Omega \rightarrow \mathbb{R} \text { measurable }: T_{k}(u) \in W^{1, p}(\Omega) \text { for all } k>0\right\} .
$$

It is possible to give meaning to the derivative $D u$ of a function $u \in \mathcal{T}_{l o c}^{1,1}(\Omega)$, generalizing the usual concept of weak derivative in $W_{l o c}^{1,1}(\Omega)$, thanks to the following result (see [B-V, Lemma 2.1]):

"For every $u \in \mathcal{T}_{\text {loc }}^{1,1}(\Omega)$ there exists a unique measurable function $v: \Omega \rightarrow \mathbb{R}$ such that

$$
D T_{k}(u)=v 1_{\{|u|<k\}} \quad \text { a.e. }
$$

Furthermore, if $u \in W_{l o c}^{1,1}(\Omega)$ then $v=D u$ in the usual weak sense."

The derivative $D u$ of a function $u \in \mathcal{T}_{\text {loc }}^{1,1}(\Omega)$ is defined as the unique function $v$ satisfying (2.1). This notation will be used throughout in the sequel.

Let $\Omega$ be a bounded open subset of $\mathbb{R}^{N}$ of class $C^{1}$, and $1 \leq p<\infty$. It is wellknown (cf. $[\mathrm{N}]$ or $[\mathrm{M}]$ ) that if $u \in W^{1, p}(\Omega)$, it is possible to define the trace of $u$ on $\partial \Omega$. More precisely, there exists a bounded operator $\gamma$ from $W^{1, p}(\Omega)$ into $L^{p}(\partial \Omega)$ such that $\gamma(u)=u_{\mid \partial \Omega}$ whenever $u \in C(\bar{\Omega})$. Now, it is easy to see that, in general, it is not possible to define the trace of an element of $\mathcal{T}^{1, p}(\Omega)$. In dimension one it is enough to consider the function $u(x)=1 / x$ for $x \in] 0,1[$. Nevertheless, we are going to define the trace for the elements of a subset $\mathcal{T}_{t r}^{1, p}(\Omega)$ of $\mathcal{T}^{1, p}(\Omega) . \mathcal{T}_{t r}^{1, p}(\Omega)$ will be the subset of $\mathcal{T}^{1, p}(\Omega)$ consisting of the functions that can be approximated by functions of $W^{1, p}(\Omega)$ in the following sense: a function $u \in \mathcal{T}^{1, p}(\Omega)$ belongs to $\mathcal{T}_{t r}^{1, p}(\Omega)$ if there exists a sequence $u_{n} \in W^{1, p}(\Omega)$ such that

(a) $u_{n} \rightarrow u$ a.e. in $\Omega$,

(b) $D T_{k}\left(u_{n}\right) \rightarrow D T_{k}(u)$ in $L^{1}(\Omega)$ for any $k>0$,

(c) the sequence $\left\{\gamma\left(u_{n}\right)\right\}$ converges a.e. in $\partial \Omega$.

Obviously, we have

$$
W^{1, p}(\Omega) \subset \mathcal{T}_{\text {tr }}^{1, p}(\Omega) \subset \mathcal{T}^{1, p}(\Omega)
$$


In (2.2) the inclusions are strict. In fact: It is easy to see that the function $u(x)=$ $1 / x$ for $x \in] 0,1\left[\right.$ is an element of $\mathcal{T}^{1,1}(] 0,1[) \sim \mathcal{T}_{t r}^{1,1}(] 0,1[)$. Moreover the function $u$ defined by

$$
u(x):= \begin{cases}1 / x & \text { if } x \in] 0,1[ \\ -1 / x & \text { if } x \in]-1,0[,\end{cases}
$$

is an example of an element of $\mathcal{T}_{t r}^{1,1}(]-1,1[) \sim W^{1,1}(]-1,1[)$.

In the following result ([AMST, Theorem 3.1]) we obtain an extension of the trace defined in $W^{1, p}(\Omega)$.

Theorem 2.1. Let $\Omega$ be a bounded open subset of $\mathbb{R}^{N}$ of class $C^{1}$, and $1 \leq p<\infty$. Then, there exists a map $\tau: \mathcal{T}_{t r}^{1, p}(\Omega) \rightarrow M(\partial \Omega, \mu)$ such that

$$
\tau(u)=\gamma(u) \quad \text { whenever } u \in W^{1, p}(\Omega) .
$$

Moreover,

(i) $\gamma\left(T_{k} u\right)=T_{k}(\tau u)$ for every $u \in \mathcal{T}_{t r}^{1, p}(\Omega)$ and $k>0$.

(ii) If $u \in \mathcal{T}_{\text {tr }}^{1, p}(\Omega)$ and $\phi \in W^{1, p}(\Omega) \cap L^{\infty}(\Omega)$, then $u-\phi \in \mathcal{T}_{t r}^{1, p}(\Omega)$ and $\tau(u-\phi)=\tau(u)-\tau(\phi)$.

To study the Dirichlet problem, in [B-V] was introduced the subspace $\mathcal{T}_{0}^{1, p}(\Omega)$ of $\mathcal{T}^{1, p}(\Omega)$ consisting of the functions that can be approximated by smooth functions with compact support in $\Omega$ in the following sense: a function $u \in \mathcal{T}^{1, p}(\Omega)$ belongs to $\mathcal{T}_{0}^{1, p}(\Omega)$ if for every $k>0$ there exists a sequence $\zeta_{n} \in C_{0}^{\infty}(\Omega)$ such that

$$
\begin{gathered}
\zeta_{n} \rightarrow T_{k} u \quad \text { in } L_{l o c}^{1}(\Omega), \\
D \zeta_{n} \rightarrow D T_{k}(u) \quad \text { in } L^{p}(\Omega) .
\end{gathered}
$$

As a consequence of the characterizations of $\mathcal{T}_{0}^{1, p}(\Omega)$ given in [B-V, Appendix II] we have

$$
\operatorname{Ker}(\tau)=\mathcal{T}_{0}^{1, p}(\Omega)
$$

We refer the reader to $[\mathrm{Ba}],[\mathrm{Be}],[\mathrm{BCP}]$ and $[\mathrm{Cr}]$ for background material on the theory of nonlinear semigroups.

\section{The CASE OF BOUNDED $\Omega$}

Throughout this section $\Omega$ is a bounded domain in $\mathbb{R}^{N}(N \geq 2)$ with smooth boundary $\partial \Omega$ of class $C^{1}, 1<p<N$, a is a vector valued mapping from $\Omega \times \mathbb{R}^{N}$ into $\mathbb{R}^{N}$ satisfying $\left(\mathrm{H}_{1}\right)-\left(\mathrm{H}_{3}\right)$, and $\beta$ is a maximal monotone graph in $\mathbb{R} \times \mathbb{R}$ with $0 \in \beta(0)$.

In this section we establish existence and uniqueness of solutions of the non-linear parabolic equation with nonlinear boundary condition

$$
\begin{gathered}
u_{t}=\operatorname{div} \mathbf{a}(x, D u) \quad \text { in } Q_{T}=(0, T) \times \Omega, \\
-\frac{\partial u}{\partial \eta_{a}} \in \beta(u) \quad \text { on } S_{T}=(0, T) \times \partial \Omega, \\
u(x, 0)=u_{0}(x) \quad \text { in } \Omega,
\end{gathered}
$$

for every initial datum in $L^{1}(\Omega)$. 
In [AMST] we associate a completely accretive operator in $L^{1}(\Omega)$ with the formal differential expresion

$$
-\operatorname{div} \mathbf{a}(x, D u)+\text { non-linear boundary conditions. }
$$

To define it we need to introduce the following subset of $W^{1, p}(\Omega)$ : Given $\beta$, a maximal monotone graph in $\mathbb{R} \times \mathbb{R}$ with $0 \in \beta(0)$, we set

$$
W_{\beta}^{1, p}(\Omega):=\left\{u \in W^{1, p}(\Omega): u(x) \in \mathcal{D}(\beta) \text { a.e. } x \in \partial \Omega\right\} .
$$

The above definition uses the fact that the trace of $u \in W^{1,1}(\Omega)$ on $\partial \Omega$ is well defined in $L^{1}(\partial \Omega)$ [N, Theorem 4.2]. Observe that we use the same notation $u$ for $u$ and its trace when convenient.

Notice that if $\mathcal{D}(\beta)$ is closed then $W_{\beta}^{1, p}(\Omega)$ is a closed convex subset of $W^{1, p}(\Omega)$. When $\beta$ corresponds to the Dirichlet boundary condition, $W_{\beta}^{1, p}(\Omega)=W_{0}^{1, p}(\Omega)$, and when $\beta$ corresponds to the Neumann boundary condition, $W_{\beta}^{1, p}(\Omega)=W^{1, p}(\Omega)$.

We define the operator $A$ in $L^{1}(\Omega)$ by the rule:

$(u, v) \in A$ if and only if $u \in W^{1, p}(\Omega) \cap L^{\infty}(\Omega), v \in L^{1}(\Omega)$, there exists $w \in$ $L^{1}(\partial \Omega)$ with $-w(x) \in \beta(u(x))$ a.e. on $\partial \Omega$ and

$$
\int_{\Omega}\langle\mathbf{a}(x, D u), D(u-\phi)\rangle \leq \int_{\Omega} v(u-\phi)+\int_{\partial \Omega} w(u-\phi)
$$

for every $\phi \in W_{\beta}^{1, p}(\Omega) \cap L^{\infty}(\Omega)$.

To characterize the closure of the operator $A$ we define the operator $\mathcal{A}$ in $L^{1}(\Omega)$ by the rule:

$(u, v) \in \mathcal{A}$ if and only if $u, v \in L^{1}(\Omega), u \in \mathcal{T}_{t r}^{1, p}(\Omega)$ and there exists $w \in L^{1}(\partial \Omega)$ with $-w(x) \in \beta(u(x))$ a.e. on $\partial \Omega$ such that

$$
\int_{\Omega}\left\langle\mathbf{a}(x, D u), D T_{k}(u-\phi)\right\rangle \leq \int_{\Omega} v T_{k}(u-\phi)+\int_{\partial \Omega} w T_{k}(u-\phi)
$$

for every $\phi \in W_{\beta}^{1, p}(\Omega) \cap L^{\infty}(\Omega)$ and $k>0$.

Notice that the integrals in (3.2) are well defined. In general, the difference of two elements of $\mathcal{T}^{1, p}(\Omega)$ is not an element of $\mathcal{T}^{1, p}(\Omega)$ (see [B-V]); however, since $\phi \in W^{1, p}(\Omega) \cap L^{\infty}(\Omega)$, we have $u-\phi \in \mathcal{T}_{t r}^{1, p}(\Omega)$ (Theorem 2.1). Hence, $T_{k}(u-\phi) \in W^{1, p}(\Omega) \cap L^{\infty}(\Omega)$, and consequently the two first integrals in (3.2) are well defined. Moreover, in the last integral we can use the fact that the trace of $f \in W^{1, p}(\Omega)$ on $\partial \Omega$ is well defined in $L^{p}(\partial \Omega)$.

Also we need to recall the following definition due to Ph. Bénilan (see [AMST]). We say that $\mathbf{a}$ is smooth if for every $f \in L^{\infty}(\Omega)$ there exists $g \in L^{1}(\partial \Omega)$ such that the solution $u$ of the Dirichlet problem

$$
\begin{gathered}
-\operatorname{div} \mathbf{a}(x, D u)=f \quad \text { in } \Omega, \\
u=0 \quad \text { on } \partial \Omega,
\end{gathered}
$$

is a solution of the Neumann problem

$$
\begin{gathered}
-\operatorname{div} \mathbf{a}(x, D u)=f \quad \text { in } \Omega, \\
\frac{\partial u}{\partial \eta_{a}}=g \quad \text { on } \partial \Omega .
\end{gathered}
$$

In the following theorem we summarize all the results we need about the operators $A$ and $\mathcal{A}$ given in [AMST]. 
Theorem 3.1. Assume that $\mathcal{D}(\beta)$ is closed or $\mathbf{a}$ is smooth. Then, the operator $A$ is completely accretive, $L^{\infty}(\Omega) \subset R(I+A)$ and $\overline{\mathcal{D}(A)}=L^{1}(\Omega)$. Moreover, $\mathcal{A}$ is the closure of $A$ in $L^{1}(\Omega)$. Consequently, $\mathcal{A}$ is an m-completely accretive operator in $L^{1}(\Omega)$ with dense domain.

We transcribe (I) as the evolution problem in $L^{1}(\Omega)$ :

$$
\frac{d u}{d t}+\mathcal{A} u \ni 0, \quad u(0)=u_{0} .
$$

By Theorem 3.1, according to the Crandall-Liggett Generation Theorem, for every initial datum $u_{0} \in L^{1}(\Omega)$ there exists a unique mild solution $u \in C\left(0, T ; L^{1}(\Omega)\right)$ of the evolution problem (II), with $u(t)=S(t) u_{0}$, where $(S(t))_{t \geq 0}$ is the semigroup of order-preserving contractions given by the exponential formula

$$
S(t) u_{0}=\lim _{n \rightarrow \infty}\left(I+\frac{t}{n} \mathcal{A}\right)^{-n} u_{0}
$$

Moreover, since $\mathcal{A}$ is completely accretive, if the initial datum $u_{0} \in \mathcal{D}(\mathcal{A})$ then the mild solution $u(t)=S(t) u_{0}$ is a strong solution (see [BCr-2]), i.e.,

$$
u \in W^{1,1}\left(0, T ; L^{1}(\Omega)\right)
$$

and (II) is satisfied almost everywhere. The next result is a consequence of nonlinear semigroup theory. We include the proof here for the sake of completeness.

Lemma 3.2. Assume that $\mathcal{D}(\beta)$ is closed or a is smooth. Let $u_{0} \in \mathcal{D}(A)$ and let $u(t)=S(t) u_{0}$ be the mild solution of $(I I)$. Then $u \in L^{p}\left(0, T ; W_{\beta}^{1, p}(\Omega)\right) \cap$ $W^{1,1}\left(0, T ; L^{1}(\Omega)\right)$ for every $T>0$, and there exists $w \in L^{1}\left(S_{T}\right)$ with $-w(t, x) \in$ $\beta(u(t, x))$ a.e. on $S_{T}$ such that

$$
\int_{\Omega}\langle\mathbf{a}(x, D u(t)), D(u(t)-\phi)\rangle \leq-\int_{\Omega} u^{\prime}(t)(u(t)-\phi)+\int_{\partial \Omega} w(t)(u(t)-\phi)
$$

for every $\phi \in W_{\beta}^{1, p}(\Omega) \cap L^{\infty}(\Omega)$ and a.e. on $[0, T]$.

Proof. Since $u(t)=S(t) u_{0}$ is a strong solution of (II), the set $K$ consisting of those values of $t \in[0, T]$ for which either $u$ is not differentiable at $t$, or $t$ is not a Lebesgue point for $u^{\prime}$, or $u^{\prime}(t)+A u(t) \not \supset 0$, is a null subset of $[0, T]$. Then, since $u^{\prime} \in L^{1}\left(0, T ; L^{1}(\Omega)\right)$, [BCP, Proposition 1.5] guarantees us that for each $\epsilon>0$, there exists a partition $0=t_{0}<t_{1}<\ldots<t_{n-1} \leq T<t_{n}$ with the properties that $t_{k} \notin K, k=1, \ldots, n, t_{k}-t_{k-1}<\epsilon$ for $k=1, \ldots, n$, and

$$
\sum_{k=1}^{n} \int_{t_{k-1}}^{t_{k}}\left\|u^{\prime}(s)-u^{\prime}\left(t_{k}\right)\right\| d s<\epsilon .
$$

If one defines $v_{\epsilon}$ as $v_{\epsilon}(0):=u_{0}, v_{\epsilon}(t):=u\left(t_{k}\right)$ on $\left.] t_{k-1}, t_{k}\right], k=1, \ldots, n$, then $v_{\epsilon}$ is a solution of an $\epsilon$-discretization of (II), and consequently $v_{\epsilon} \rightarrow u$ in $C\left(0, T ; L^{1}(\Omega)\right)$.

Since $\left(u\left(t_{k}\right),-u^{\prime}\left(t_{k}\right)\right) \in A$, there exists $w_{k} \in L^{1}(\partial \Omega)$ with $-w_{k}(x) \in \beta\left(u\left(t_{k}, x\right)\right)$ a.e. on $\partial \Omega$, such that

$$
\int_{\Omega}\left\langle\mathbf{a}\left(x, D u\left(t_{k}\right)\right), D\left(u\left(t_{k}\right)-\phi\right)\right\rangle \leq-\int_{\Omega} u^{\prime}\left(t_{k}\right)\left(u\left(t_{k}\right)-\phi\right)+\int_{\partial \Omega} w_{k}\left(u\left(t_{k}\right)-\phi\right)
$$


for every $\phi \in W_{\beta}^{1, p}(\Omega) \cap L^{\infty}(\Omega)$. From here, if we set $w_{\epsilon}(t):=w_{k}$ and $u_{\epsilon}(t):=u^{\prime}\left(t_{k}\right)$ on $\left.] t_{k-1}, t_{k}\right], k=1, \ldots, n$, we get

$$
\int_{0}^{T} \int_{\Omega}\left\langle\mathbf{a}\left(x, D v_{\epsilon}\right), D\left(v_{\epsilon}-\phi\right)\right\rangle \leq-\int_{0}^{T} \int_{\Omega} u_{\epsilon}\left(v_{\epsilon}-\phi\right)+\int_{0}^{T} \int_{\partial \Omega} w_{\epsilon}\left(v_{\epsilon}-\phi\right)
$$

for every $\phi \in L^{\infty}\left(Q_{T}\right) \cap L^{p}\left(0, T ; W_{\beta}^{1, p}(\Omega)\right)$. Taking $\phi=0$ as test function in (3.6), by $\left(\mathrm{H}_{1}\right)$ and $(3.4)$ we have

$$
\begin{aligned}
& \int_{0}^{T} \int_{\Omega}\left|D v_{\epsilon}\right|^{p} \leq-\int_{0}^{T} \int_{\Omega} u_{\epsilon} v_{\epsilon}+\int_{0}^{T} \int_{\partial \Omega} w_{\epsilon} v_{\epsilon} \\
& \quad \leq-\int_{0}^{T} \int_{\Omega} u_{\epsilon} v_{\epsilon} \leq\left\|u_{\epsilon}\right\|_{L^{1}\left(Q_{T}\right)}\left\|u_{0}\right\|_{\infty} \leq M\left\|u_{0}\right\|_{\infty} .
\end{aligned}
$$

From this it follows that $\left\{\left|D v_{\epsilon}\right|: 0<\epsilon<1\right\}$ is a bounded subset of $L^{p}\left(Q_{T}\right)$. Hence, after passing to a suitable subsequence, we have

$$
D v_{\epsilon} \rightarrow v \in L^{p}\left(Q_{T}\right)^{N} \quad \text { weakly in } L^{p}\left(Q_{T}\right)^{N} \text { as } \epsilon \rightarrow 0 .
$$

Now, since $v_{\epsilon} \rightarrow u$ in $C\left(0, T ; L^{1}(\Omega)\right)$, we have $v=D u$. Thus, it follows from $v_{\epsilon} \in L^{p}\left(0, T ; W_{\beta}^{1, p}(\Omega)\right)$ that $u \in L^{p}\left(0, T ; W_{\beta}^{1, p}(\Omega)\right)$.

On the other hand, since $\left(u(t),-u^{\prime}(t)\right) \in A$, there exists $w(t) \in L^{1}(\partial \Omega)$ with $-w(t)(x) \in \beta(u(t)(x))$ a.e. in $x \in \partial \Omega$, such that

$$
\int_{\Omega}\langle\mathbf{a}(x, D u(t)), D(u(t)-\phi)\rangle \leq-\int_{\Omega} u^{\prime}(t)(u(t)-\phi)+\int_{\partial \Omega} w(t)(u(t)-\phi)
$$

for every $\phi \in W_{\beta}^{1, p}(\Omega) \cap L^{\infty}(\Omega)$. Taking $\phi=u(t)-T_{k}\left(u(t)-v_{\epsilon}(t)\right)$ in the above inequality, we get

$$
\begin{aligned}
& \int_{\Omega}\left\langle\mathbf{a}(x, D u(t)), D T_{k}\left(u(t)-v_{\epsilon}(t)\right)\right\rangle \\
& \quad \leq-\int_{\Omega} u^{\prime}(t) T_{k}\left(u(t)-v_{\epsilon}(t)\right)+\int_{\partial \Omega} w(t) T_{k}\left(u(t)-v_{\epsilon}(t)\right) .
\end{aligned}
$$

Taking $\phi=v_{\epsilon}(t)-T_{k}\left(v_{\epsilon}(t)-u(t)\right)$ as test function in (3.5), we get

$$
\begin{aligned}
& \int_{\Omega}\left\langle\mathbf{a}\left(x, D v_{\epsilon}(t)\right), D T_{k}\left(v_{\epsilon}(t)-u(t)\right)\right\rangle \\
& \quad \leq-\int_{\Omega} u_{\epsilon}(t) T_{k}\left(v_{\epsilon}(t)-u(t)\right)+\int_{\partial \Omega} w_{\epsilon}(t) T_{k}\left(v_{\epsilon}(t)-u(t)\right) .
\end{aligned}
$$

Adding (3.8) and (3.9), we obtain

$$
\begin{aligned}
0 \leq & \int_{\Omega}\left\langle\mathbf{a}(x, D u(t))-\mathbf{a}\left(x, D v_{\epsilon}(t)\right), D T_{k}\left(u(t)-v_{\epsilon}(t)\right)\right\rangle \\
& \leq-\int_{\Omega}\left(u^{\prime}(t)-u_{\epsilon}(t)\right) T_{k}\left(u(t)-v_{\epsilon}(t)\right)+\int_{\partial \Omega}\left(w(t)-w_{\epsilon}(t)\right) T_{k}\left(u(t)-v_{\epsilon}(t)\right) .
\end{aligned}
$$

From this it follows that

$$
\int_{\partial \Omega}\left|w(t)-w_{\epsilon}(t)\right| \leq \int_{\Omega}\left|u^{\prime}(t)-u_{\epsilon}(t)\right| .
$$

Finally, from (3.4) and (3.10), we get $w \in L^{1}\left(S_{T}\right)$, and the proof concludes. 
The above theorem motivates us to give the following definition of a solution of the problem

$$
\begin{gathered}
u_{t}=\operatorname{div} \mathbf{a}(x, D u) \quad \text { in } Q_{T}=\Omega \times(0, T), \\
-\frac{\partial u}{\partial \eta_{a}} \in \beta(u) \quad \text { on } S_{T}=\partial \Omega \times(0, T) .
\end{gathered}
$$

Definition. A measurable function $u: Q_{T} \rightarrow \mathbb{R}$ is an entropy solution of (III) in $Q_{T}$ if $u \in C\left(0, T ; L^{1}(\Omega)\right), u(t) \in \mathcal{T}_{t r}^{1, p}(\Omega)$ for almost all $t \in[0, T], T_{k} u \in$ $L^{p}\left(0, T ; W_{\beta}^{1, p}(\Omega)\right)$ for all $k>0$ and there exists $w \in L^{1}\left(S_{T}\right)$ with $-w(t, x) \in$ $\beta(u(t, x))$ a.e. on $S_{T}$ such that

$$
\begin{aligned}
& \int_{0}^{t} \int_{\Omega}\left\langle\mathbf{a}(x, D u), D T_{k}(u-\phi)\right\rangle \leq-\int_{0}^{t} \int_{\Omega} \frac{\partial \phi}{\partial s} T_{k}(u-\phi) \\
& \quad+\int_{\Omega} J_{k}(u(0)-\phi(0))-\int_{\Omega} J_{k}(u(t)-\phi(t))+\int_{0}^{t} \int_{\partial \Omega} w T_{k}(u-\phi)
\end{aligned}
$$

for all $k>0$, for all $t \in[0, T]$ and $\phi \in \mathcal{T}\left(Q_{T}\right)$, where

$$
\mathcal{T}\left(Q_{T}\right):=L^{\infty}\left(Q_{T}\right) \cap L^{p}\left(0, T ; W_{\beta}^{1, p}(\Omega)\right) \cap W^{1,1}\left(0, T ; L^{1}(\Omega)\right)
$$

and

$$
J_{k}(r):=\int_{0}^{r} T_{k}(s) d s .
$$

The purpose of this section is to prove existence and uniqueness of entropy solutions for problem (I) when the initial data are in $L^{1}(\Omega)$. Moreover, we will see that these entropy solutions are the mild solutions. We start with existence.

Theorem 3.3. Assume that $\mathcal{D}(\beta)$ is closed or $\mathbf{a}$ is smooth. Let $u_{0} \in L^{1}(\Omega)$ and let $u(t)=S(t) u_{0}$ be the mild solution of (II). Then, $u$ is an entropy solution of (III) for all $T>0$.

Proof. Let $u_{n}^{0} \in \mathcal{D}(A)$ be such that $u_{n}^{0} \rightarrow u_{0}$ in $L^{1}(\Omega)$ and let $f \in L^{1}(\Omega)$ be such that $\left|u_{n}^{0}\right| \leq f$ for all $n \in \mathbb{N}$. By the above lemma, if $u_{n}(t):=S(t) u_{n}^{0}$, we have $u_{n} \in L^{p}\left(0, T ; W_{\beta}^{1, p}(\Omega)\right) \cap W^{1,1}\left(0, T ; L^{1}(\Omega)\right)$ and there exists $w_{n} \in L^{1}\left(S_{T}\right)$ with $-w_{n}(t, x) \in \beta\left(u_{n}(t, x)\right)$ a.e. on $S_{T}$ such that

$$
\begin{aligned}
& \int_{\Omega}\left\langle\mathbf{a}\left(x, D u_{n}(s)\right), D\left(u_{n}(s)-\phi\right)\right\rangle \\
& \quad \leq-\int_{\Omega} u_{n}^{\prime}(s)\left(u_{n}(s)-\phi\right)+\int_{\partial \Omega} w_{n}(s)(u(s)-\phi)
\end{aligned}
$$

for every $\phi \in W_{\beta}^{1, p}(\Omega) \cap L^{\infty}(\Omega)$ and for almost all $0<s<T$.

We introduce the class $\mathcal{F}$ of functions $S \in C^{2}(\mathbb{R}) \cap L^{\infty}(\mathbb{R})$ satisfying

$$
S(0)=0,0 \leq S^{\prime} \leq 1, S^{\prime}(s)=0 \text { for } s \text { large enough, }
$$

$$
S(-s)=-S(s), \quad \text { and } S^{\prime \prime}(s) \leq 0 \quad \text { for } s \geq 0 .
$$


Let $\mathcal{G}:=\mathcal{F} \cup\left\{T_{k}: k>0\right\}$. Then, given $\phi \in \mathcal{T}\left(Q_{T}\right)$ and $S \in \mathcal{G}$, using $u_{n}(s)-$ $S\left(u_{n}(s)-\phi(s)\right)$ as test function in (3.12), and integrating, we obtain

$$
\begin{aligned}
& \int_{0}^{t} \int_{\Omega}\left\langle\mathbf{a}\left(x, D u_{n}(s)\right), D S\left(u_{n}(s)-\phi(s)\right)\right\rangle \\
& \quad \leq-\int_{0}^{t} \int_{\Omega} u_{n}^{\prime}(s) S\left(u_{n}(s)-\phi(s)\right)+\int_{0}^{t} \int_{\partial \Omega} w_{n}(s) S\left(u_{n}(s)-\phi(s)\right)
\end{aligned}
$$

for every $\phi \in \mathcal{T}\left(Q_{T}\right), S \in \mathcal{G}$ and for all $0<t<T$.

For every $S \in \mathcal{G}$, let $J_{S}(r):=\int_{0}^{r} S(s) d s$. Then,

$$
\frac{\partial}{\partial s} J_{S}\left(u_{n}-\phi\right)=S\left(u_{n}-\phi\right) \frac{\partial}{\partial s}\left(u_{n}-\phi\right)
$$

From (3.13) and (3.14), it follows that

$$
\begin{aligned}
\int_{0}^{t} \int_{\Omega} & \left\langle\mathbf{a}\left(x, D u_{n}(s)\right), D S\left(u_{n}(s)-\phi(s)\right)\right\rangle \\
\leq & -\int_{0}^{t} \int_{\Omega} \frac{\partial \phi}{\partial s} S\left(u_{n}(s)-\phi(s)\right)+\int_{\Omega} J_{S}\left(u_{n}(0)-\phi(0)\right) \\
& -\int_{\Omega} J_{S}\left(u_{n}(t)-\phi(t)\right)+\int_{0}^{t} \int_{\partial \Omega} w_{n}(s) S\left(u_{n}(s)-\phi(s)\right)
\end{aligned}
$$

for every $\phi \in \mathcal{T}\left(Q_{T}\right), S \in \mathcal{G}$ and for all $0<t<T$.

Taking $\phi=0$ and $S=T_{k}$ in (3.15) and using $\left(\mathrm{H}_{1}\right)$, we get

$$
\begin{aligned}
\int_{0}^{t} \int_{\Omega}\left|D T_{k}\left(u_{n}(s)\right)\right|^{p} & \leq \int_{0}^{t} \int_{\Omega}\left\langle\mathbf{a}\left(x, D u_{n}(s)\right), D T_{k}\left(u_{n}(s)\right)\right\rangle \\
\leq \int_{\Omega} J_{k}\left(u_{n}(0)\right) & \leq k \int_{\Omega}\left|u_{n}^{0}\right| \leq k\|f\|_{1},
\end{aligned}
$$

consequently, $\left\{D T_{k} u_{n}\right\}_{n \in \mathbb{N}}$ is a bounded sequence in $L^{p}\left(Q_{T}\right)$. Hence, there exists a subsequence, still denoted by $D T_{k} u_{n}$, such that $D T_{k} u_{n} \rightarrow h$ weakly in $L^{p}\left(Q_{T}\right)$. Now, since $T_{k} u_{n} \rightarrow T_{k}(u)$ in $L^{p}\left(Q_{T}\right)$, it follows that $D T_{k} u_{n} \rightarrow D T_{k}(u)$ weakly in $L^{p}\left(Q_{T}\right)$.

We now prove that $\left\{D u_{n}\right\}_{n \in \mathbb{N}}$ is a Cauchy sequence in measure. To do this we follow the same technique used in [BG-1] (see also [AMST]). Let $r, \epsilon>0$. For some $A>1$, we set

$$
C(x, A, r):=\inf \{\langle\mathbf{a}(x, \xi)-\mathbf{a}(x, \eta), \xi-\eta\rangle:|\xi| \leq A,|\eta| \leq A,|\xi-\eta| \geq r\} .
$$

Having in mind that the function $\psi \rightarrow \mathbf{a}(x, \psi)$ is continuous for almost all $x \in \Omega$ and the set $\{(\xi, \eta):|\xi| \leq A,|\eta| \leq A,|\xi-\eta| \geq t\}$ is compact, we conclude that the infimum in the definition of $C(x, A, r)$ is a minimum. Hence, by $\left(\mathrm{H}_{2}\right)$, it follows that

$$
C(x, A, r)>0 \quad \text { for almost all } x \in \Omega \text {. }
$$

For $k>0$ and $n, m \in \mathbb{N}$, we have

$$
\begin{gathered}
\left\{\left|D u_{n}-D u_{m}\right|>r\right\} \subset\left\{\left|D T_{A} u_{n}\right| \geq A\right\} \cup\left\{\left|D T_{A} u_{m}\right| \geq A\right\} \cup\left\{\left|u_{n}\right|>A\right\} \\
\cup\left\{\left|u_{m}\right|>A\right\} \cup\left\{\left|u_{n}-u_{m}\right| \geq k^{2}\right\} \cup\{C(x, A, r) \leq k\} \cup G,
\end{gathered}
$$


where

$$
\begin{gathered}
G:=\left\{\left|u_{n}-u_{m}\right| \leq k^{2},\left|u_{n}\right| \leq A,\left|u_{m}\right| \leq A, C(x, A, r) \geq k\right. \\
\left.\left|D T_{A} u_{n}\right| \leq A,\left|D T_{A} u_{m}\right| \leq A,\left|D u_{n}-D u_{m}\right|>r\right\}
\end{gathered}
$$

Now,

$$
\begin{gathered}
\lambda_{N+1}\left(\left\{\left|u_{n}\right|>A\right\}\right) \leq \int_{0}^{T} \int_{\Omega} \frac{\left|u_{n}\right|}{A} \leq \frac{1}{A} \int_{0}^{T}\left\|u_{n}(t)\right\|_{L^{1}(\Omega)} \\
\leq \frac{1}{A} \int_{0}^{T}\left\|u_{n}^{0}\right\|_{L^{1}(\Omega)} \leq \frac{T}{A}\left\|u_{n}^{0}\right\|_{L^{1}(\Omega)} \leq \frac{T}{A}\|f\|_{1} .
\end{gathered}
$$

Hence, we can choose $A$ large enough to have

$$
\lambda_{N+1}\left(\left\{\left|u_{n}\right|>A\right\} \cup\left\{\left|u_{m}\right|>A\right\}\right) \leq \frac{\epsilon}{5} .
$$

On the other hand, by (3.16), we have

$$
\lambda_{N+1}\left(\left\{\left|D T_{A} u_{n}\right|>A\right\}\right) \leq \int_{0}^{T} \int_{\Omega} \frac{\left|D T_{A} u_{n}\right|^{p}}{A^{p}} \leq\|f\|_{1} A^{1-p} .
$$

Then, we can choose $A$ large enough to have

$$
\lambda_{N+1}\left(\left\{\left|D T_{A} u_{n}\right|>A\right\} \cup\left\{\left|D T_{A} u_{m}\right|>A\right\}\right) \leq \frac{\epsilon}{5} .
$$

By (3.17), taking $k$ small enough we have

$$
\lambda_{N+1}\left(\left\{(t, x) \in Q_{T}: C(x, A, r) \leq k\right\}\right) \leq \frac{\epsilon}{5} .
$$

Since $u_{n}, u_{m} \in \mathcal{T}\left(Q_{T}\right)$, on inserting the test functions $u_{n}, u_{m}$ in (3.13), and adding and dropping unnecessary positive terms, one has

$$
\begin{aligned}
\int_{0}^{T} & \int_{\Omega}\left\langle\mathbf{a}\left(x, D u_{n}(s)\right)-\mathbf{a}\left(x, D u_{m}(s)\right), D T_{k}\left(u_{n}(s)-u_{m}(s)\right)\right\rangle \\
& \leq-\int_{0}^{T} \int_{\Omega}\left(u_{n}^{\prime}(s)-u_{m}^{\prime}(s)\right) T_{k}\left(u_{n}(s)-u_{m}(s)\right) \\
& =-\int_{0}^{T} \int_{\Omega} \frac{\partial}{\partial s} J_{k}\left(u_{n}(s)-u_{m}(s)\right) \\
& =\int_{\Omega} J_{k}\left(u_{n}(0)-u_{m}(0)\right)-\int_{\Omega} J_{k}\left(u_{n}(T)-u_{m}(T)\right) \\
& \leq \int_{\Omega} J_{k}\left(u_{n}(0)-u_{m}(0)\right) \leq k \int_{\Omega}\left|u_{n}^{0}-u_{m}^{0}\right| \leq 2 k\|f\|_{1}
\end{aligned}
$$

Hence

$$
\begin{gathered}
\lambda_{N+1}(G) \\
\leq \lambda_{N+1}\left(\left\{\left|u_{n}-u_{m}\right| \leq k^{2},\left\langle\mathbf{a}\left(x, D u_{n}\right)-\mathbf{a}\left(x, D u_{m}\right), D\left(u_{n}-u_{m}\right)\right\rangle \geq k\right\}\right) \\
\leq \frac{1}{k} \int_{\left\{\left|u_{n}-u_{m}\right|<k^{2}\right\}}\left\langle\mathbf{a}\left(x, D u_{n}\right)-\mathbf{a}\left(x, D u_{m}\right), D\left(u_{n}-u_{m}\right)\right\rangle \leq \frac{2}{k} k^{2}\|f\|_{1} \leq \frac{\epsilon}{5}
\end{gathered}
$$

for $k$ small enough. 
Finally, since $A$ and $k$ have been already choosen and $\left\{u_{n}\right\}$ is a Cauchy sequence in $L^{1}\left(Q_{T}\right)$, if $n_{0}$ is large enough we have for $n, m \geq n_{0}$ the estimate

$$
\lambda_{N+1}\left(\left\{\left|u_{n}-u_{m}\right| \geq k^{2}\right\}\right) \leq \frac{\epsilon}{5}
$$

From here, using (3.18), (3.19), (3.20), (3.21) and (3.22), we can conclude that

$$
\lambda_{N+1}\left(\left\{\left|D u_{n}-D u_{m}\right| \geq r\right\}\right) \leq \epsilon \text { for } m, n \geq n_{0} .
$$

Consequently, $\left\{D u_{n}\right\}_{n \in \mathbb{N}}$ is a Cauchy sequence in measure. Now, the above argument also shows that $\left\{D T_{k} u_{n}\right\}_{n \in \mathbb{N}}$ is a Cauchy sequence in measure for every $k>0$. Moreover, since $\left\{D T_{k} u_{n}\right\}_{n \in \mathbb{N}}$ is bounded in $L^{p}\left(Q_{T}\right)$, by [B-V, Lemma 6.1], $\left\{D T_{k} u_{n}\right\}_{n \in \mathbb{N}}$ converges to $D T_{k}(u)$ in $L^{1}\left(Q_{T}\right)$. Thus,

$$
\left\{D u_{n}\right\}_{n \in \mathbb{N}} \text { converges to } D u \text { in measure. }
$$

According to Nemytskii's Theorem [K, Lemma I.2.2.1] the convergence of $D u_{n}$ to $D u$ in measure implies that $\mathbf{a}\left(x, D u_{n}\right)$ converges in measure to $\mathbf{a}(x, D u)$, and a.e. (up to extraction of a subsequence, if necessary).

We now claim that

$$
\left\{w_{n}\right\} \text { is a Cauchy sequence in } L^{1}\left(S_{T}\right) \text {. }
$$

Taking the test functions $u_{n}, u_{m}$ in (3.13), and adding and dropping unnecessary positive terms, we get

$$
\begin{gathered}
-\int_{0}^{T} \int_{\partial \Omega}\left(w_{n}-w_{m}\right) T_{k}\left(u_{n}-u_{m}\right) \leq-\int_{0}^{T} \int_{\Omega}\left(u_{n}^{\prime}-u_{m}^{\prime}\right) T_{k}\left(u_{n}-u_{m}\right) \\
=-\int_{0}^{T} \int_{\Omega} \frac{\partial}{\partial s} J_{k}\left(u_{n}-u_{m}\right)=\int_{\Omega} J_{k}\left(u_{n}^{0}-u_{m}^{0}\right)-\int_{\Omega} J_{k}\left(u_{n}(T)-u_{m}(T)\right) \\
\leq \int_{\Omega} J_{k}\left(u_{n}^{0}-u_{m}^{0}\right) \leq k \int_{\Omega}\left|u_{n}^{0}-u_{m}^{0}\right| .
\end{gathered}
$$

Dividing by $k$ and letting $k \rightarrow 0$, we see that

$$
\int_{0}^{T} \int_{\partial \Omega}\left|w_{n}-w_{m}\right| \leq \int_{\Omega}\left|u_{n}^{0}-u_{m}^{0}\right| .
$$

Consequently, the claim (3.24) holds and there exists $w \in L^{1}\left(S_{T}\right)$ such that

$$
w_{n} \rightarrow w \text { in } L^{1}\left(S_{T}\right) .
$$

Let us see now that $u(t) \in \mathcal{T}_{t r}^{1, p}(\Omega)$ for almost all $t \in[0, T]$. Indeed: Let

$$
A_{k}:=\left\{(t, x) \in S_{T}:\left|T_{k} u(t)(x)\right|<k\right\},
$$

and

$$
C:=S_{T} \sim \bigcup_{k=1}^{\infty} A_{k}
$$

Then, for every $k>0$, we have

$$
\begin{aligned}
\mu(C) & =\frac{1}{k} \int_{C}\left|T_{k} u\right| \leq \frac{1}{k} \int_{S_{T}}\left|T_{k} u\right| \leq \frac{C_{1}}{k} \int_{0}^{T}\left\|T_{k} u(t)\right\|_{W^{1,1}(\Omega)} \\
& \leq \frac{C_{2}}{k}\left(\int_{0}^{T}\left\|T_{k} u(t)\right\|_{L^{1}(\Omega)}+\int_{0}^{T}\left\|D T_{k} u(t)\right\|_{L^{p}(\Omega)}\right) .
\end{aligned}
$$


Now,

$$
\left\|T_{k} u(t)\right\|_{L^{1}(\Omega)} \leq\|u(t)\|_{L^{1}(\Omega)} \leq\|u(0)\|_{L^{1}(\Omega)} .
$$

Moreover, by (3.16) we have

$$
\begin{aligned}
\int_{0}^{T}\left\|D T_{k} u(t)\right\|_{L^{p}(\Omega)} & =\int_{0}^{T}\left(\int_{\Omega}\left|D T_{k} u(t)\right|^{p}\right)^{1 / p} \\
& \leq C_{3}\left(\int_{0}^{T} \int_{\Omega}\left|D T_{k} u(t)\right|^{p}\right)^{1 / p} \leq C_{4} k^{1 / p} .
\end{aligned}
$$

Hence,

$$
\mu(C) \leq \frac{C_{2}}{k}\left(\|u(0)\|_{L^{1}(\Omega)}+C_{4} k^{1 / p}\right) \quad \text { for any } k>0 .
$$

Taking the limit as $k \rightarrow \infty$, we have $\mu(C)=0$. Moreover, $A_{k} \subset A_{r}$ if $k \leq r$. Thus, the function $v$ in $S_{T}$ given by

$$
v(t, x):=\left(T_{k} u(t)\right)(x) \quad \text { if }(t, x) \in A_{k}
$$

is well defined.

On the other hand, since $C$ is a null subset of $S_{T}$, there exists a null subset $B$ of $[0, T]$ such that the sections $C_{t}=\{x \in \partial \Omega:(t, x) \in C\}$ are null subsets of $\partial \Omega$ for all $t \notin B$. For every $t \notin B$, we define in $\Omega$ the function $v_{n}(t):=T_{n}(u(t))$. Then, $v_{n}(t) \in W^{1, p}(\Omega)$ for all $n \in \mathbb{N}$ and $v_{n}(t) \rightarrow u(t)$ as $n \rightarrow \infty$ a.e. in $\Omega$. Moreover, for every $k>0$,

$$
D T_{k}\left(v_{n}(t)\right) \rightarrow D T_{k}(u(t)) \quad \text { in } L^{1}(\Omega) .
$$

Finally, if $x \notin C_{t}$, there is $n_{0} \in \mathbb{N}$ such that $(t, x) \in A_{n}$ for all $n \geq n_{0}$. Hence, $v(t, x)=\left(T_{n} u(t)\right)(x)=v_{n}(t)(x)$ for all $n \geq n_{0}$. Thus, $v_{n}(t) \rightarrow v(t)$ as $n \rightarrow \infty$ a.e. in $\partial \Omega$. Consequently, $u(t) \in \mathcal{T}_{t r}^{1, p}(\Omega)$ for all $t \notin B$.

We now claim that, up to extraction of a subsequence,

$$
u_{n} \rightarrow v \quad \text { a.e. in } S_{T} .
$$

In fact, since

$$
\int_{0}^{T}\left\|T_{k} u_{n}(t)-T_{k} u(t)\right\|_{L^{1}(\partial \Omega)} \leq M \int_{0}^{T}\left\|T_{k} u_{n}(t)-T_{k} u(t)\right\|_{W^{1,1}(\Omega)},
$$

we have

$$
T_{k} u_{n} \rightarrow T_{k} u \quad \text { in } L^{1}\left(S_{T}\right) \quad \text { as } n \rightarrow \infty .
$$

Then, by a diagonal process, we can find a subsequence $u_{n_{j}}$ and a null subset $D \subset S_{T}$ such that

$$
\left(T_{k} u_{n_{j}}(t)\right)(x) \rightarrow\left(T_{k} u(t)\right)(x) \quad \text { as } j \rightarrow \infty \quad \text { for }(t, x) \in S_{T} \sim D .
$$

From here it is easy to see that

$$
u_{n_{j}}(t, x) \rightarrow v(t, x) \quad \text { as } j \rightarrow \infty \quad \text { for any }(t, x) \in S_{T} \sim(C \cup D),
$$

and the claim (3.26) holds. 
To complete the proof it remains to show that $-w(t, x) \in \beta(u(t, x))$ a.e. on $S_{T}$ and that for every $\phi \in \mathcal{T}\left(Q_{T}\right), k>0$ and all $t \in[0, T]$

$$
\begin{aligned}
& \int_{0}^{t} \int_{\Omega}\left\langle\mathbf{a}(x, D u), D T_{k}(u-\phi)\right\rangle \leq-\int_{0}^{t} \int_{\Omega} \frac{\partial \phi}{\partial s} T_{k}(u-\phi) \\
& \quad+\int_{\Omega} J_{k}(u(0)-\phi(0))-\int_{\Omega} J_{k}(u(t)-\phi(t))+\int_{0}^{t} \int_{\partial \Omega} w T_{k}(u-\phi) .
\end{aligned}
$$

Suppose first that $S \in \mathcal{F}$ and $\phi \in \mathcal{T}\left(Q_{T}\right)$. Then, by (3.15) we have

$$
\begin{aligned}
\int_{0}^{t} \int_{\Omega}\left\langle\mathbf{a}\left(x, D u_{n}(s)\right), D S\left(u_{n}(s)-\phi(s)\right)\right\rangle \\
\leq-\int_{0}^{t} \int_{\Omega} \frac{\partial \phi}{\partial s} S\left(u_{n}(s)-\phi(s)\right)+\int_{\Omega} J_{S}\left(u_{n}(0)-\phi(0)\right) \\
\quad-\int_{\Omega} J_{S}\left(u_{n}(t)-\phi(t)\right)+\int_{0}^{t} \int_{\partial \Omega} w_{n}(s) S\left(u_{n}(s)-\phi(s)\right) .
\end{aligned}
$$

We can write the first member of (3.28) as

$$
\begin{aligned}
\int_{0}^{t} & \int_{\Omega}\left\langle\mathbf{a}\left(x, D u_{n}(s)\right), D u_{n}(s)\right\rangle S^{\prime}\left(u_{n}(s)-\phi(s)\right) \\
& -\int_{0}^{t} \int_{\Omega}\left\langle\mathbf{a}\left(x, D u_{n}(s)\right), D \phi(s)\right\rangle S^{\prime}\left(u_{n}(s)-\phi(s)\right) .
\end{aligned}
$$

Since $u_{n} \rightarrow u$ and $D u_{n} \rightarrow D u$ a.e., we have by Fatou's lemma

$$
\begin{gathered}
\int_{0}^{t} \int_{\Omega}\langle\mathbf{a}(x, D u(s)), D u(s)\rangle S^{\prime}(u(s)-\phi(s)) \\
\leq \liminf _{n \rightarrow \infty} \int_{0}^{t} \int_{\Omega}\left\langle\mathbf{a}\left(x, D u_{n}(s)\right), D u_{n}(s)\right\rangle S^{\prime}\left(u_{n}(s)-\phi(s)\right) .
\end{gathered}
$$

The second term of (3.29) is estimated as follows. Let $r:=\|\phi\|_{\infty}+\|S\|_{\infty}$. By (3.16) and $\left(\mathrm{H}_{3}\right)$, it follows that

$$
\left\{\mathbf{a}\left(x, D T_{r} u_{n}\right): n \in \mathbb{N}\right\} \quad \text { is bounded in } L^{p^{\prime}}\left(Q_{T}\right) .
$$

Then, up to extraction of a subsequence, we can suppose that

$$
\mathbf{a}\left(x, D T_{r} u_{n}\right) \rightarrow \mathbf{a}\left(x, D T_{r} u\right) \quad \text { weakly in } L^{p^{\prime}}\left(Q_{T}\right) .
$$

On the other hand,

$$
\left|D \phi S^{\prime}\left(u_{n}-\phi\right)\right| \leq M|D \phi| \in L^{p}\left(Q_{T}\right) .
$$

Then, by the Dominated Convergence Theorem, we have

$$
D \phi S^{\prime}\left(u_{n}-\phi\right) \rightarrow D \phi S^{\prime}(u-\phi) \quad \text { in } L^{p}\left(Q_{T}\right)^{N} .
$$

Hence, by (3.30) and (3.31), it follows that

$$
\begin{gathered}
\lim _{n \rightarrow \infty} \int_{0}^{t} \int_{\Omega}\left\langle\mathbf{a}\left(x, D u_{n}(s)\right), D \phi(s)\right\rangle S^{\prime}\left(u_{n}(s)-\phi(s)\right) \\
=\int_{0}^{t} \int_{\Omega}\langle\mathbf{a}(x, D u(s)), D \phi(s)\rangle S^{\prime}(u(s)-\phi(s)) .
\end{gathered}
$$


Therefore, applying again the Dominated Convergence Theorem in the second member of (3.28), we obtain

$$
\begin{gathered}
\int_{0}^{t} \int_{\Omega}\langle\mathbf{a}(x, D u), D S(u-\phi)\rangle \leq-\int_{0}^{t} \int_{\Omega} \frac{\partial \phi}{\partial s} S(u-\phi) \\
+\int_{\Omega} J_{S}(u(0)-\phi(0))-\int_{\Omega} J_{S}(u(t)-\phi(t))+\int_{0}^{t} \int_{\partial \Omega} w S(u-\phi) .
\end{gathered}
$$

From here, to get (3.27) we only need to apply the technique used in the proof of [B-V, Lemma 3.2].

Finally, since $-w_{n} \in \beta\left(u_{n}\right)$ a.e. on $S_{T}$, by (3.25), (3.26) and the closedness of $\beta$, we get $-w \in \beta(u)$ a.e. on $S_{T}$.

In order to prove the uniqueness of entropy solutions we give first the following result.

Lemma 3.4. Assume that $\mathcal{D}(\beta)$ is closed or $\mathbf{a}$ is smooth. Let $u_{0} \in L^{1}(\Omega)$ and $v_{0} \in$ $\mathcal{D}(A)$. Let $u(t)$ be the entropy solution with initial datum $u_{0}$ and let $v(t)=S(t) v_{0}$ be the mild solution with initial datum $v_{0}$. Then, for every $k>0$ and $t \in[0, T]$ we have

In particular,

$$
\int_{\Omega} J_{k}(u(t)-v(t)) \leq \int_{\Omega} J_{k}\left(u_{0}-v_{0}\right)
$$

$$
\int_{\Omega}|u(t)-v(t)| \leq \int_{\Omega}\left|u_{0}-v_{0}\right|
$$

Proof. By Lemma 3.2, there exists $\hat{w} \in L^{1}\left(S_{T}\right)$ with $-\hat{w}(t, x) \in \beta(v(t, x))$ a.e. on $S_{T}$, such that

$$
\begin{aligned}
& \int_{\Omega}\left\langle\mathbf{a}(x, D v(t)), D T_{k}(v(t)-\phi)\right\rangle \\
& \quad \leq-\int_{\Omega} v^{\prime}(t) T_{k}(v(t)-\phi)+\int_{\partial \Omega} \hat{w}(t) T_{k}(v(t)-\phi)
\end{aligned}
$$

for every $\phi \in W_{\beta}^{1, p}(\Omega) \cap L^{\infty}(\Omega), k>0$ and almost all $0<t<T$. Now, since $u$ is an entropy solution, for any $h>0, T_{h} u(t) \in W_{\beta}^{1, p}(\Omega) \cap L^{\infty}(\Omega)$. Hence, taking $T_{h} u(s)$ as test function in (3.32) and integrating, we get

$$
\begin{aligned}
& \int_{0}^{t} \int_{\Omega}\left\langle\mathbf{a}(x, D v(s)), D T_{k}\left(v(s)-T_{h} u(s)\right)\right\rangle \\
& \quad \leq-\int_{0}^{t} \int_{\Omega} v^{\prime}(s) T_{k}\left(v(s)-T_{h} u(s)\right)+\int_{0}^{t} \int_{\partial \Omega} \hat{w}(s) T_{k}\left(v(s)-T_{h} u(s)\right) .
\end{aligned}
$$

On the other hand, taking $\phi=v(s)$ as test function in the definition of entropy solution, we have that there exists $w \in L^{1}\left(S_{T}\right)$ with $-w(t, x) \in \beta(u(t, x))$ a.e. on $S_{T}$ such that

$$
\begin{aligned}
\int_{0}^{t} \int_{\Omega}\left\langle\mathbf{a}(x, D u(s)), D T_{k}(u(s)-v(s))\right\rangle \leq-\int_{0}^{t} \int_{\Omega} \frac{\partial v}{\partial s} T_{k}(u(s)-v(s)) \\
\quad+\int_{\Omega} J_{k}(u(0)-v(0))-\int_{\Omega} J_{k}(u(t)-v(t))+\int_{0}^{t} \int_{\partial \Omega} w(s) T_{k}(u(s)-v(s)) .
\end{aligned}
$$


Now,

$$
\begin{gathered}
\int_{0}^{t} \int_{\Omega}\left\langle\mathbf{a}(x, D v(s)), D T_{k}\left(v(s)-T_{h} u(s)\right)\right\rangle+\int_{0}^{t} \int_{\Omega}\left\langle\mathbf{a}(x, D u(s)), D T_{k}(u(s)-v(s))\right\rangle \\
=\iint_{\{|u| \leq h\}}\left\langle\mathbf{a}(x, D u(s))-\mathbf{a}(x, D v(s)), D T_{k}(u(s)-v(s))\right\rangle \\
+\iint_{\{|u|>h\}}\left\langle\mathbf{a}(x, D v(s)), D T_{k}(v(s)-h \operatorname{sign} u(s))\right\rangle \\
+\iint_{\{|u|>h\}}\left\langle\mathbf{a}(x, D u(s)), D T_{k}(u(s)-v(s))\right\rangle \\
\geq \iint_{\{|u|>h\}}\left\langle\mathbf{a}(x, D u(s)), D T_{k}(u(s)-v(s))\right\rangle=0
\end{gathered}
$$

if $h>k+\left\|v_{0}\right\|_{\infty}$.

On the other hand, having in mind that $u(s) \in \mathcal{T}_{t r}^{1, p}(\Omega)$, by the Dominated Convergence Theorem it follows that

$$
\lim _{h \rightarrow \infty} \int_{0}^{t} \int_{\Omega} v^{\prime}(s)\left(T_{k}\left(v(s)-T_{h} u(s)\right)-T_{k}(v(s)-u(s))\right)=0
$$

and

$$
\lim _{h \rightarrow \infty} \int_{0}^{t} \int_{\partial \Omega} \hat{w}(s) T_{k}\left(v(s)-T_{h} u(s)\right)=\int_{0}^{t} \int_{\partial \Omega} \hat{w}(s) T_{k}(v(s)-u(s)) .
$$

Therefore, since

$$
\int_{0}^{t} \int_{\partial \Omega} \hat{w}(s) T_{k}(v(s)-u(s))+\int_{0}^{t} \int_{\partial \Omega} w(s) T_{k}(u(s)-v(s)) \leq 0,
$$

adding (3.33) and (3.34), we get

$$
\int_{\Omega} J_{k}(u(t)-v(t)) \leq \int_{\Omega} J_{k}\left(u_{0}-v_{0}\right)
$$

Finally, since

$$
\lim _{k \rightarrow 0} \frac{J_{k}(r)}{k}=|r|,
$$

by the Dominated Convergence Theorem we get

$$
\int_{\Omega}|u(t)-v(t)| \leq \int_{\Omega}\left|u_{0}-v_{0}\right|
$$

Theorem 3.5. Assume that $\mathcal{D}(\beta)$ is closed or $\mathbf{a}$ is smooth. Let $u_{0} \in L^{1}(\Omega)$. Then, the entropy solution $u(t)$ of problem (III) with initial datum $u_{0}$ is unique and coincides with the mild solution $v(t)=S(t) u_{0}$.

Proof. Take $u_{n}^{0} \in \mathcal{D}(A)$ such that $u_{n}^{0} \rightarrow u_{0}$ in $L^{1}(\Omega)$. By the above lemma,

$$
\int_{\Omega}\left|S(t) u_{n}^{0}-u(t)\right| \leq \int_{\Omega}\left|u_{n}^{0}-u_{0}\right|
$$

for all $t \in[0, T]$. Then, since $S(t) u_{n}^{0} \rightarrow v(t)$ in $L^{1}(\Omega)$, we get $u(t)=v(t)$. 
To finish this section we will see that every entropy solution of (III) is a weak solution and has some regularity properties which are optimal in the particular case of the heat equation. The method used in the proof was suggested by Ph. Bénilan.

Theorem 3.6. Assume that $\mathcal{D}(\beta)$ is closed or $\mathbf{a}$ is smooth. Let $u_{0} \in L^{1}(\Omega)$. Then, the entropy solution $u(t)$ of problem (III) with initial datum $u_{0}$ is a weak solution of (III), i.e.,

$$
u_{t}=\operatorname{div} \mathbf{a}(x, D u) \quad \text { in } \mathcal{D}^{\prime}\left(Q_{T}\right) .
$$

Moreover,

$$
u \in M^{p_{1}}\left(Q_{T}\right), \quad|D u| \in M^{p_{2}}\left(Q_{T}\right),
$$

where $p_{1}=p-1+\frac{p}{N}$ and $p_{2}=\frac{N(p-1)+p}{N+1}$. In case $p>1+\frac{N}{N+1}, u \in L^{q}\left(0, T ; W^{1, q}(\Omega)\right)$ for every $1 \leq q<p_{2}$.

Proof. Take $u_{n}^{0} \in \mathcal{D}(A)$ such that $u_{n}^{0} \rightarrow u_{0}$ in $L^{1}(\Omega)$. Let $f \in L^{1}(\Omega)$ be such that $\left|u_{n}^{0}\right| \leq f$ a.e. in $\Omega$ for all $n \in \mathbb{N}$. For every $n \in \mathbb{N}$, let $u_{n}(t):=S(t) u_{n}^{0}$. By Theorem 3.5 , we have $u(t)=S(t) u_{0}$. Since $u_{n} \rightarrow u$ in $L^{1}\left(Q_{T}\right)$, there exists $g \in L^{1}\left(Q_{T}\right)$ such that $\left|u_{n}\right| \leq g$ a.e. in $Q_{T}$ for all $n \in \mathbb{N}$.

We claim that

$$
\left\{u_{n}\right\}_{n \in \mathbb{N}} \text { is bounded in } M^{p_{1}}\left(Q_{T}\right), \quad p_{1}=p-1+\frac{p}{N} .
$$

Let $p^{\star}=\frac{p N}{N-p}$. By Poincaré's inequality (cf. [Zi, Chapter 4]) and since $\left|u_{n}(t)\right| \leq g$, there exist constants $C_{i}>0$ such that

$$
\begin{gathered}
\left\|T_{k} u_{n}(t)\right\|_{p^{\star}}^{p} \leq\left(\left\|T_{k} u_{n}(t)-\overline{T_{k} u_{n}(t)}\right\|_{p^{\star}}+\left\|\overline{T_{k} u_{n}(t)}\right\|_{p^{\star}}\right)^{p} \\
\leq\left(C_{1}\left\|D T_{k} u_{n}(t)\right\|_{p}+\left\|\overline{T_{k} u_{n}(t)}\right\|_{p^{\star}}\right)^{p} \leq C_{2}\left(\left\|D T_{k} u_{n}(t)\right\|_{p}+\left\|T_{k} u_{n}(t)\right\|_{1}\right)^{p} \\
\leq C_{3}\left(1+\left\|D T_{k} u_{n}(t)\right\|_{p}\right)^{p} .
\end{gathered}
$$

Then, by (3.16), it follows that

$$
\begin{gathered}
\int_{0}^{T}\left\|T_{k} u_{n}(t)\right\|_{p^{\star}}^{p} d t \leq C_{3} \int_{0}^{T}\left(1+\left\|D T_{k} u_{n}(t)\right\|_{p}\right)^{p} d t \\
\leq C_{4}\left(1+\int_{0}^{T}\left\|D T_{k} u_{n}(t)\right\|_{p}^{p} d t\right) \leq C_{4}(1+M k) .
\end{gathered}
$$

Thus

$$
\int_{0}^{T}\left(\lambda_{N}\left(\left\{\left|u_{n}(t)\right| \geq k\right\}\right)\right)^{p / p^{\star}} d t \leq \int_{0}^{T}\left(\frac{1}{k}\left\|T_{k} u_{n}(t)\right\|_{p^{\star}}\right)^{p} d t \leq \frac{C_{5}(1+k)}{k^{p}} .
$$

Moreover

$$
\lambda_{N}\left(\left\{\left|u_{n}(t)\right| \geq k\right\}\right) \leq \frac{1}{k}\left\|u_{n}(t)\right\|_{1} \leq \frac{C_{6}}{k} .
$$

Then, by (3.37) and (3.38), we have

$$
\lambda_{N+1}\left(\left\{\left|u_{n}\right| \geq k\right\}\right)=\int_{0}^{T} \lambda_{N}\left(\left\{\left|u_{n}(t)\right| \geq k\right\}\right) d t
$$




$$
\begin{gathered}
=\int_{0}^{T}\left(\lambda_{N}\left(\left\{\left|u_{n}(t)\right| \geq k\right\}\right)\right)^{1-p / p^{\star}}\left(\lambda_{N}\left(\left\{\left|u_{n}(t)\right| \geq k\right\}\right)\right)^{p / p^{\star}} d t \\
\leq\left(\frac{C_{6}}{k}\right)^{1-p / p^{\star}} \int_{0}^{T}\left(\lambda_{N}\left(\left\{\left|u_{n}(t)\right| \geq k\right\}\right)\right)^{p / p^{\star}} d t \\
\leq C_{7} \frac{1}{k^{1-p / p^{\star}}} \frac{(1+k)}{k^{p}} \leq \frac{C_{8}}{k^{p-p / p^{\star}}}=C_{8} k^{-p_{1}} .
\end{gathered}
$$

Next, we claim that

$$
\left\{D u_{n}\right\}_{n \in \mathbb{N}} \text { is bounded in } M^{p_{2}}\left(Q_{T}\right), \quad p_{2}=\frac{N(p-1)+p}{N+1} .
$$

Let $r>0$. By (3.16), there exists a constant $Q_{1}>0$ such that for every $k>0$ and $n \in \mathbb{N}$,

$$
\lambda_{N+1}\left(\left\{\left|D T_{k}\left(u_{n}\right)\right|>r / 2\right\}\right) \leq \int_{0}^{T} \int_{\Omega} \frac{\left|D T_{k}\left(u_{n}\right)\right|^{p}}{(r / 2)^{p}} \leq \frac{Q_{1} k}{r^{p}} .
$$

On the other hand, by (3.36), there exists a constant $Q_{2}>0$ such that

$$
\lambda_{N+1}\left(\left\{\left|u_{n}\right| \geq k\right\}\right) \leq \frac{Q_{2}}{k^{p_{1}}} \quad \text { for every } k>0 \text { and } n \in \mathbb{N} .
$$

From (3.40) and (3.41), it follows that

$$
\begin{gathered}
\lambda_{N+1}\left(\left\{\left|D u_{n}\right|>r\right\}\right) \\
\leq \lambda_{N+1}\left(\left\{\left|D u_{n}-D T_{k}\left(u_{n}\right)\right|>r / 2\right\}\right)+\lambda_{N+1}\left(\left\{\left|D T_{k}\left(u_{n}\right)\right|>r / 2\right\}\right) \\
\leq \lambda_{N+1}\left(\left\{\left|u_{n}\right| \geq k\right\}\right)+\lambda_{N+1}\left(\left\{\left|D T_{k}\left(u_{n}\right)\right|>r / 2\right\}\right) \leq \frac{Q_{2}}{k^{p_{1}}}+\frac{Q_{1} k}{r^{p}} .
\end{gathered}
$$

Then, taking $k:=r^{p_{2} / p_{1}}$, we have

$$
\lambda_{N+1}\left(\left\{\left|D u_{n}\right|>r\right\}\right) \leq Q r^{-p_{2}} \text { for every } n \in \mathbb{N},
$$

and the claim (3.39) is satisfied.

From (3.23), (3.36) and (3.39) we can state that

$$
u \in M^{p_{1}}\left(Q_{T}\right), \quad|D u| \in M^{p_{2}}\left(Q_{T}\right),
$$

where $p_{1}=p-1+\frac{p}{N}$ and $p_{2}=\frac{N(p-1)+p}{N+1}$. Suppose we are in the case $p>1+\frac{N}{N+1}$. Then $p_{2}>1$. Hence, if $1 \leq q<p_{2}$, we have that $u \in L^{q}\left(Q_{T}\right)$ and $D u \in L^{q}\left(Q_{T}\right)$. Consequently, $u \in L^{q}\left(0, T ; W^{1, q}(\Omega)\right)$ for every $1 \leq q<p_{2}$.

Let us see now that $u$ is a weak solution of (III). Indeed: By (3.39), $\left\{\left|D u_{n}\right|: n \in\right.$ $\mathbb{N}\}$ is a bounded sequence in $M^{p_{2}}\left(Q_{T}\right)$. Thus, $\left\{\left|D u_{n}\right|^{p-1}: n \in \mathbb{N}\right\}$ is a bounded sequence in $M^{q_{1}}\left(Q_{T}\right)$ with $q_{1}=1+\frac{1}{(p-1)(N+1)}$. Then, by $\left(\mathrm{H}_{3}\right)$ it follows that $\left\{\left|\mathbf{a}\left(x, D u_{n}\right)\right|: n \in \mathbb{N}\right\}$ is a bounded sequence in $M^{q_{1}}\left(Q_{T}\right)$. Hence, if $1 \leq q<q_{1}$, $\left\{\left|\mathbf{a}\left(x, D u_{n}\right)\right|: n \in \mathbb{N}\right\}$ is a bounded sequence in $L^{q}\left(Q_{T}\right)$. Moreover, by the proof of Theorem 3.3, we know that

$$
\mathbf{a}\left(x, D u_{n}\right) \rightarrow \mathbf{a}(x, D u) \quad \text { in measure. }
$$

Then, by [B-V, Lemma 6.1],

$$
\mathbf{a}\left(x, D u_{n}\right) \rightarrow \mathbf{a}(x, D u) \quad \text { in } L^{1}\left(Q_{T}\right) .
$$


On the other hand, since each $u_{n}$ is a strong solution and $u_{n} \in L^{\infty}\left(Q_{T}\right)$, given $\phi \in \mathcal{D}\left(Q_{T}\right)$, if we take $u_{n}+\phi$ and $u_{n}-\phi$ as test functions in (3.11), we obtain

$$
\int_{0}^{T} \int_{\Omega}\left\langle\mathbf{a}\left(x, D u_{n}\right), D \phi\right\rangle=-\int_{0}^{T} \int_{\Omega} \frac{\partial u_{n}}{\partial t} \phi=\int_{0}^{T} \int_{\Omega} \frac{\partial \phi}{\partial t} u_{n} .
$$

Letting $n \rightarrow \infty$ in the last equality, we get

$$
\int_{0}^{T} \int_{\Omega}\langle\mathbf{a}(x, D u), D \phi\rangle=\int_{0}^{T} \int_{\Omega} \frac{\partial \phi}{\partial t} u
$$

for all $\phi \in \mathcal{D}\left(Q_{T}\right)$.

\section{The Case of a Dirichlet boundary condition for general $\Omega$}

From now on $\Omega$ is an open set, not necessarily bounded, in $\mathbb{R}^{N}(N \geq 2), 1<p<$ $N$, and a is a vector valued mapping from $\Omega \times \mathbb{R}^{N}$ into $\mathbb{R}^{N}$ satisfying $\left(\mathrm{H}_{1}\right)-\left(\mathrm{H}_{3}\right)$. In this section we establish existence and uniqueness of solutions of the initial-value problem for the non-linear parabolic equation with Dirichlet boundary condition

$$
\begin{gathered}
u_{t}=\operatorname{div} \mathbf{a}(x, D u) \quad \text { in } Q_{T}=(0, T) \times \Omega, \\
u=0 \quad \text { on } S_{T}=(0, T) \times \partial \Omega, \\
u(x, 0)=u_{0}(x) \quad \text { in } \Omega,
\end{gathered}
$$

for every initial datum in $L^{1}(\Omega)$.

We use the following completely accretive operators introduced in $[\mathrm{B}-\mathrm{V}]$ instead of the operators $A$ and $\mathcal{A}$ of the previous section.

We define the operator $B$ in $L^{1}(\Omega)$ by the rule: $(u, v) \in B$ if and only if $u \in$ $\mathcal{T}_{0}^{1, p}(\Omega) \cap L^{\infty}(\Omega) \cap L^{1}(\Omega), v \in L^{1}(\Omega)$ and $-\operatorname{div} \mathbf{a}(x, D u)=v$ in $\mathcal{D}^{\prime}(\Omega)$.

The closure of the operator $B$ in $L^{1}(\Omega)$ is the operator $\mathcal{B}$ defined by the rule: $(u, v) \in \mathcal{B}$ if and only if $u, v \in L^{1}(\Omega), u \in \mathcal{T}_{0}^{1, p}(\Omega)$ and

$$
\int_{\Omega}\left\langle\mathbf{a}(x, D u), D T_{k}(u-\phi)\right\rangle \leq \int_{\Omega} v T_{k}(u-\phi)
$$

for every $\phi \in \mathcal{T}_{0}^{1, p}(\Omega) \cap L^{\infty}(\Omega)$ and $k>0$.

In the following theorem we summarize all the results we need about the operators $B$ and $\mathcal{B}$ given in $[\mathrm{B}-\mathrm{V}]$.

Theorem 4.1. The operator $B$ is completely accretive, $L^{\infty}(\Omega) \subset R(I+B)$ and $\overline{\mathcal{D}(B)}=L^{1}(\Omega)$. Moreover, $\mathcal{B}$ is the closure of $B$ in $L^{1}(\Omega)$. Consequently, $\mathcal{B}$ is an $m$-completely accretive operator in $L^{1}(\Omega)$ with dense domain.

We transcribe (IV) as the evolution problem in $L^{1}(\Omega)$

$$
\frac{d u}{d t}+\mathcal{B} u=0, \quad u(0)=u_{0} .
$$

By Theorem 4.1, according to the Crandall-Liggett Generation Theorem, for every initial datum $u_{0} \in L^{1}(\Omega)$ there exists a unique mild solution $u \in C\left(0, T ; L^{1}(\Omega)\right)$ of the evolution problem $(\mathrm{V})$, with $u(t)=T(t) u_{0}$, where $(T(t))_{t \geq 0}$ is the semigroup of order-preserving contractions given by the exponential formula

$$
T(t) u_{0}=\lim _{n \rightarrow \infty}\left(I+\frac{t}{n} \mathcal{B}\right)^{-n} u_{0}
$$


As in the bounded case, we want to characterize the mild solutions as weak solutions satisfying certain entropy inequality. To do that we introduce the following definition of solution for the problem

$$
u_{t}=\operatorname{div} \mathbf{a}(x, D u) \quad \text { in } Q_{T}=\Omega \times(0, T),
$$

$$
u=0 \quad \text { on } S_{T}=\partial \Omega \times(0, T) .
$$

Definition. A measurable function $u: Q_{T} \rightarrow \mathbb{R}$ is an entropy solution of (VI) in $Q_{T}$ if $u \in C\left(0, T ; L^{1}(\Omega)\right), u(t) \in \mathcal{T}_{0}^{1, p}(\Omega)$ for almost all $t \in[0, T], D T_{k} u \in L^{p}\left(Q_{T}\right)$ for all $k>0$ and

$$
\begin{aligned}
& \int_{0}^{t} \int_{\Omega}\left\langle\mathbf{a}(x, D u), D T_{k}(u-\phi)\right\rangle \\
& \quad \leq-\int_{0}^{t} \int_{\Omega} \frac{\partial \phi}{\partial s} T_{k}(u-\phi)+\int_{\Omega} J_{k}(u(0)-\phi(0))-\int_{\Omega} J_{k}(u(t)-\phi(t)),
\end{aligned}
$$

for all $k>0, t \in[0, T]$ and $\phi \in L^{\infty}\left(Q_{T}\right) \cap W^{1,1}\left(0, T ; L^{1}(\Omega)\right)$, with $\phi(t) \in \mathcal{T}_{0}^{1, p}(\Omega)$ for almost all $t$ and $D \phi \in L^{p}\left(Q_{T}\right)$.

Using the same technique as in the bounded case and small modifications in the proofs of the theorems of $\S 3$, we get existence and uniqueness of entropy solutions for problem (IV) when the initial data are in $L^{1}(\Omega)$. Concretely, we can establish the following result.

Theorem 4.2. For every initial datum $u_{0} \in L^{1}(\Omega)$, the mild solution $u(t)=$ $T(t) u_{0}$ of problem $(V)$ is the unique entropy solution of problem (VI). Moreover, $u(t)$ is a weak solution of (VI) and

$$
u \in M^{p_{1}}\left(Q_{T}\right), \quad|D u| \in M^{p_{2}}\left(Q_{T}\right),
$$

where $p_{1}=p-1+\frac{p}{N}$ and $p_{2}=\frac{N(p-1)+p}{N+1}$. In case $p>1+\frac{N}{N+1}, u \in L^{q}\left(0, T ; W_{l o c}^{1, q}(\Omega)\right)$ for every $1 \leq q<p_{2}$.

\section{ACKNOWLEDGEMENTS}

The authors gratefully acknowledge the many helpful suggestions of Ph. Bénilan during the preparation of the paper.

Added in proof. The referee has point out to us the existence of the paper $\mathrm{X}$. Xu, On the initial-boundary-valued problem for $u_{t}-\operatorname{div}\left(|\nabla u|^{p-2} \nabla u\right)=0$, Arch. Rat. Mech. Anal. 127 (1994), pp. 319-335 (MR 95j:35115), in which renormalized solutions are introduced for the particular case of the $p$-Laplacian with Dirichlet boundary condition. Existence and uniqueness of this type of solutions are proved. It is not difficult to see that this concept and the concept of entropy solution introduced here coincide.

\section{REFERENCES}

[AMST] F. Andreu, J. M. Mazón, S. Segura de León and J. Toledo, Quasi-linear elliptic and parabolic equations in $L^{1}$ with non-linear boundary conditions, Adv. Math. Sci. Appl. 7 (1997), 183-213. MR 98f:35079

[Ba] V. Barbu, Nonlinear Semigroups and Differential Equations in Banach Spaces, Noordhoff, Leyden, 1976. MR 52:11166 
[Be] Ph. Bénilan, Equations d'évolution dans un espace de Banach quelconque et applications, Thèse Orsay, 1972.

[B-V] Ph. Bénilan, L. Boccardo, Th. Gallouët, R. Gariepy, M. Pierre and J. L. Vazquez, An $L^{1}$-Theory of Existence and Uniqueness of Solutions of Nonlinear Elliptic Equations, Ann. Scuola Norm. Sup. Pisa Cl. Sci. (4) 22 (1995), 241-273. MR 96k:35052

[BBC] Ph. Bénilan, H. Brezis and M. G. Crandall, A semilinear equation in $L^{1}$, Ann. Scuola Norm. Sup. Pisa Cl. Sci. (4) 2 (1975), 523-555. MR 52:11299

[BCr-1] Ph. Bénilan and M. G. Crandall, Regularizing effects of homogeneous evolution equations, in Contribution to Analysis and Geometry (D. N. Clark et al., eds.), Johns Hopkins University Press, 1981, pp. 23-39. MR 83g:47063

[BCr-2] Ph. Bénilan and M. G. Crandall, Completely accretive operators, in Semigroup Theory and Evolution Equations (Ph. Clement et al., eds.), Marcel Dekker, 1991, pp. 41-76. MR 93e: 47071

[BCP] Ph. Bénilan, M. G. Crandall and A. Pazy, Evolution Equations Governed by Accretive Operators, Forthcoming.

[BCS] Ph. Bénilan, M. G. Crandall and P. Sacks, Some $L^{1}$ Existence and Dependence Results for Semilinear Elliptic Equations under Nonlinear Boundary Conditions, Appl. Math. Optim. 17 (1988), 203-224. MR 89d:35055

[BG-1] L. Boccardo and Th. Gallouët, Non-linear Elliptic and Parabolic Equations Involving Measure Data, J. Funct. Anal. 87 (1989), 149-169. MR 92d:35286

[BG-2] L. Boccardo and Th. Gallouët, Nonlinear elliptic equations with right-hand side measures, Comm. in Partial Diff. Equations 17 (1992), 641-655. MR 94c:35083

[Br-1] H. Brézis, Problèmes Unilatéraux, J. Math. Pures et Appl. 51 (1972), 1-168. MR 55:1166

[Br-2] H. Brézis, Opérateur maximaux monotone et semi-groupes de contractions dans les espaces de Hilbert, North-Holland, 1973. MR 50:1060

[Cr] M. G. Crandall, Nonlinear Semigroups and Evolution Governed by Accretive Operators, Proc. Symposia in Pure Math., vol. 45, Amer. Math. Soc., 1986, pp. 305-336. MR 87h: 47140

[Di-1] E. Di Benedetto, Degenerate Parabolic Equations, Springer-Verlag, 1993. MR 94h:35130

[Di-2] E. Di Benedetto, Degenerate and singular parabolic equations, in Recent Advances in Partial Differential Equations (M. A. Herrero and E. Zuazua eds., eds.), Wiley-Masson, 1994, pp. 55-84. MR 95a:35078

[DiH-1] E. Di Benedetto and M. A. Herrero, On the Cauchy problem and initial traces for a degenerate parabolic equations, Trans. Amer. Math. Soc. 314 (1989), 187-224. MR 90d:35139

[DiH-2] E. Di Benedetto and M. A. Herrero, Non-negative Solutions of the Evolution p-Laplacian Equation. Initial Traces and Cauchy problem when $1<p<2$, Arch. Rat. Mech. Anal. 111 (1990), 225-290. MR 92g:35088

[DH] J. I. Diaz and M. A. Herrero, Estimates on the support of the solutions of some nonlinear elliptic and parabolic problems, Proc. Royal Soc. Edinburgh 89A (1981), 249-258. MR 83i:35019

[DL] G. Duvaut and J. L. Lions, Inequalities in Mechanics and Physics, Springer-Verlag, 1976. MR 58:25191

[Fr] A. Friedman, Generalized Heat Transfer between Solids and Gases under Nonlinear Boundary Conditions, J. Math. Mech. 51 (1959), 161-183. MR 21:1138

[KS] D. Kinderlehrer and G. Stampacchia, An Introduction to Variational Inequalities and their Applications, Academic Press, 1980. MR 81g:49013

[K] M. A. Krasnosel'skii, Topological Methods in the Theory of Nonlinear Integral Equations, Pergamon Press, 1964. MR 28:2414

[L] J. L. Lions, Quelques méthodes de résolution de problémes aux limites non linéaires, Dunod/Gauthier-Vilars, 1968. MR 41:4326

[M] C. B. Morrey, Jr., Multiple Integrals in the Calculus of Variations, Springer-Verlag, 1966. MR 34:2380

[N] J. Nečas, Les Méthodes Directes en Théorie des Equations Elliptiques, Masson et Cie, Paris, 1967. MR 37:3168

[Ra-1] J. M. Rakotoson, Some Quasilinear Parabolic Equations, Nonlinear Analysis T. M. A. 17 (1991), 1163-1175. MR 93a:35074 
[Ra-2] J. M. Rakotoson, A Compactness Lemma for Quasilinear Problems: Application to Parabolic Equations, J. Funct. Anal. 106 (1992), 358-374. MR 94a:35067

[X] $\quad \mathrm{X} . \mathrm{Xu}, A$-Laplacian problem in $L^{1}$ with nonlinear boundary conditions, Commun. in Partial Differential Equations 19 (1994), 143-176. MR 95b:35075

[Zi] W. P. Ziemer, Weakly Differentiable Functions, Springer-Verlag, 1989. MR 91e:46046

Departamento de Análisis Matemático, Universitat de València, 46100 Burjassot, VALENCIA, SPAin

E-mail address: Fuensanta.Andreu@uv.es

E-mail address: Mazon@uv.es

E-mail address: Sergio.Segura@uv.es

E-mail address: Jose.Toledo@uv.es 